\title{
Perilla Oil Alleviates High-Fat Diet-Induced Inflammation in the Colon of Mice by Suppressing Nuclear Factor-Kappa B Activation
}

\author{
Shalom Sara Thomas, ${ }^{1}$ Youn-Soo Cha, ${ }^{1,2}$ and Kyung-Ah Kim ${ }^{3}$ \\ ${ }^{I}$ Department of Food Science and Human Nutrition, Jeonbuk National University, Jeonju, Republic of Korea. \\ ${ }^{2}$ Obesity Research Center, Jeonbuk National University, Jeonju, Republic of Korea. \\ ${ }^{3}$ Department of Food and Nutrition, Chungnam National University, Daejeon, Republic of Korea.
}

\begin{abstract}
This study aimed to evaluate the effect of perilla oil (PO) on high-fat diet (HD)-induced colonic inflammation. Male C57BL/6J mice (5 weeks old) were divided into four groups: normal diet, HD, HD supplemented with fish oil (FO), and HD supplemented with PO, and were fed experimental diets for 16 weeks. PO significantly ameliorated $(P<.05)$ the HD-induced colon inflammation as indicated by the increased colon length and low macroscopic score. PO increased the number of Bifidobacteria and reduced the number of Enterobacteriaceae, which in turn resulted in the lowering of endotoxin levels. Proinflammatory cytokines in serum and colon such as interleukin (IL)- $1 \beta$, IL-6, and tumor necrosis factor- $\alpha$ were also decreased by PO treatment. In addition, PO suppressed the expression of cyclooxygenase 2 and inducible nitric oxide, and inhibited the activation of nuclear factor- $\kappa \mathrm{B}$ in the colon while increasing the expression of the tight junction protein, Zonula occludens-1. The gene expression of GPR120, a membrane receptor activated by omega-3 fatty acids, was increased in the oiltreated groups. Altogether, PO improved HD-induced colon inflammatory conditions, and the effects were similar to those of FO, confirming that PO is a potential omega-3 fatty acid source for dietary supplements.
\end{abstract}

KEYWORDS: cytokines $\bullet$ high-fat diet $\bullet$ inflammation $\bullet$ omega-3 fatty acids

\section{INTRODUCTION}

$\mathbf{O}$ BESITY AND ITS RELATED metabolic complications are known to induce inflammation. It is characterized by the chronic production of inflammatory factors in a lowgrade state. ${ }^{1}$ The occurrence of inflammatory bowel disease (IBD) is increasing across the globe, especially in newly industrialized countries, owing to the westernization of society. ${ }^{2}$ Furthermore, a recent report revealed that the prevalence of IBD in Korea is growing steadily, and the affected population is shifting toward young adults (19-30 years old). ${ }^{3}$

Kim et al. previously showed that high-fat diet (HD) alters the gut microbial population resulting in increased endotoxin levels. Endotoxins, such as lipopolysaccharide (LPS), contribute to the onset of inflammation through the induction of Toll-like receptors, which activate nuclear factor $-\kappa \mathrm{B}(\mathrm{NF}-\kappa \mathrm{B})$ by phosphorylating inhibitor $\kappa \mathrm{B}(\mathrm{I} \kappa \mathrm{B})$. The activated NF- $\kappa \mathrm{B}$ further turns on the expression of its downstream inflammatory markers, which results in the development of chronic inflammatory conditions. ${ }^{4}$ Therefore, to improve the complications of inflammatory conditions, such as colitis, functional foods that can inhibit these

Manuscript received 30 December 2019. Revision accepted 5 May 2020.

Address correspondence to: Kyung-Ah Kim, PhD, Dept. of Food and Nutrition, Chungnam National University, 99, Daehak-ro (St), Yoseong-gu, Daejeon, Daejeon 34134, Republic of Korea, E-mail: kakim@cnu.ac.kr inflammatory signaling pathways may be potential candidates for treating inflammatory diseases.

The $\alpha$-linolenic acid, present in the seeds and nuts of plants, acts as a precursor for the synthesis of docosahexaenoic acid (DHA) and eicosapentaenoic acid (EPA), the two omega-3 fatty acids that have beneficial effects on infant development and for preventing Alzheimer's disease. ${ }^{5}$ Omega- 3 fatty acids have been shown to reduce the risk of cardiovascular disease and also improve some forms of cancer in clinical trials ${ }^{6,7}$ Fish oil (FO) is a marine source of omega- 3 fatty acids and is reported to have anti-inflammatory effects. ${ }^{8-10}$ In human beings, FO supplementation is shown to induce anti-inflammatory gene expression in immune cells. ${ }^{11}$

Perilla oil (PO), a rich source of omega-3 fatty acids, is an edible oil extracted from the seeds of Perilla frutescens used for cooking in countries such as Korea and Japan, and is known to have several health benefits. Among different plant oils, perilla seed oil is reported to have the highest amount of omega- 3 fatty acids (ranging from $54 \%$ to $60 \%$ ), and this is in the form of ALA, the precursor of EPA and DHA. It has been shown that after a considerable period of PO consumption, the levels of DHA and EPA were increased in elderly subjects. ${ }^{12}$ In addition, balancing the ratio of omega- 6 to omega-3 fatty acids is recommended for the normal functioning of many physiological actions including immune function. The ratio of omega- 6 to omega- 3 fatty acids in PO is 1:5. It adds to the nutritional importance of PO 
that it contains phenolic compounds such as rosmarinic acid, flavonoids, and anthocyanins. ${ }^{13}$

Several studies have revealed that PO has cardioprotective, antiatherosclerotic, and hypolipidemic effects. ${ }^{12,14,15} \mathrm{It}$ is also reported to improve allergic inflammation in mice. ${ }^{16}$ Compared with the extensive use of $\mathrm{PO}$ used for edible purposes, its impact on various health aspects has not yet thoroughly analyzed. Furthermore, most of the studies related to polyunsaturated fatty acid (PUFA) have focused on FO, which is used as an omega-3 fatty acid supplement. Therefore, this study aimed to evaluate the effect of PO on HD-induced colonic inflammation and thus encourage the consumption of $\mathrm{PO}$ as a supplement for omega-3 fatty acids.

\section{MATERIALS AND METHODS}

\section{Experimental diet}

Diet ingredients were purchased from DooYeol Bio (Seoul, South Korea). FO was purchased from Sigma Aldrich (St. Louis, Missouri), and a widely used commercially available PO purchased from a local supermarket. The experimental diets were prepared every week by modifying a diet composition previously described to make the diets, so that the treated group consumes $8 \%$ oil diet. ${ }^{17}$ The ingredients used in the diet are given in Table 1. Composition of fatty acids of the oils is given in Supplementary Table S1. The ratio of saturated fatty acids to unsaturated fatty acids in the diets was as follows: normal diet (ND)-0.34, HD-0.61, high-fat diet supplemented with FO (HDFO)-0.56, and highfat diet supplemented with PO (HDPO)-0.44. In addition, omega- 6 to omega- 3 ratio was $4.9,12.5,1.33$, and 1 for $\mathrm{ND}$, $\mathrm{HD}, \mathrm{HDFO}$, and HDPO, respectively.

Table 1. Components of the Experimental Diet

\begin{tabular}{lcccc}
\hline Ingredient $(g)$ & $N D$ & $H D$ & $H D F O$ & $H D P O$ \\
\hline Casein & 200 & 200 & 200 & 200 \\
L-cysteine & 3 & 3 & 3 & 3 \\
Corn starch & 506.2 & 0 & 0 & 0 \\
Maltodextrin & 125 & 125 & 125 & 125 \\
Sucrose & 68.8 & 68.8 & 68.8 & 68.8 \\
Cellulose & 50 & 50 & 50 & 50 \\
Soybean oil & 25 & 25 & 25 & 25 \\
Lard & 20 & 245 & 182 & 182 \\
Dicalcium & 13 & 13 & 13 & 13 \\
Calcium carbonate & 5.5 & 5.5 & 5.5 & 5.5 \\
Potassium citrate & 16.5 & 16.5 & 16.5 & 16.5 \\
Mineral mix & 10 & 10 & 10 & 10 \\
Vitamin mix & 10 & 10 & 10 & 10 \\
Choline bitartrate & 2 & 2 & 2 & 2 \\
FO & - & - & 63 & - \\
PO & - & - & - & 63 \\
Total (g) & 1055 & 773.8 & 773.8 & 773.8 \\
\hline
\end{tabular}

$\mathrm{ND}, 10 \% \mathrm{kcal}$ from fat; HD, $60 \% \mathrm{kcal}$ from fat; HDFO, $60 \% \mathrm{kcal}$ from fat diet supplemented with FO; HDPO, 60\% kcal from fat diet supplemented with PO. The supplemented group consumed a diet with a final composition of $8 \%$ oil in it.

FO, fish oil; HD, high-fat diet; HDFO, high-fat diet supplemented with FO; HDPO, high-fat diet supplemented with PO; ND, normal diet; PO, perilla oil.

\section{Animals and treatment}

Five-week old, male C57BL/6J mice were purchased from Chung-ang Bio (Seoul, South Korea). After 1 week of adaptation, the mice were divided into four groups: ND, HD, HDFO, and HDPO. Body weight was measured once a week and feed intake twice a week. A controlled housing environment of $23 \pm 1^{\circ} \mathrm{C}$ was used to maintain the animals, with alternate $12 \mathrm{~h}$ dark and light cycles. The guidelines of Animal Care and Use Committee of Chungnam National University (CNU-00918) were followed while conducting animal experiments.

\section{Sample collection}

After 16 weeks of experiment, the mice were euthanized with ether after $12 \mathrm{~h}$ of fasting. Portions of the tissues were frozen in liquid nitrogen and stored at $-72^{\circ} \mathrm{C}$ until analysis, and some portions were fixed in formalin for histological analyses. Colon macroscopic scores were evaluated based on a previously explained method. ${ }^{18}$ After dissecting the colon, edema or ulceration level was scored based on the following scale: no ulcer or inflammation- 0 , ulceration accompanied by local hyperemia-1, ulceration but hyperemia2 , ulceration and inflammation only at one site-3, two or more sites of ulceration and inflammation- 4 , and ulceration extending $>2 \mathrm{~cm}$ of the colon-5.

\section{Blood biochemical parameters}

Serum triglyceride (TG) and total cholesterol (TC) levels were measured using kits (ASAN Bio, Seoul, South Korea). The serum and colon levels of interleukin (IL)- $1 \beta$, IL-6, and tumor necrosis factor- $\alpha$ (TNF- $\alpha$ ) were analyzed using the kits provided by R\&D systems (Minneapolis, MN). Serum endotoxin levels were analyzed using Pierce LAL Chromogenic Endotoxin Quantitation Kit (88282; Thermo Fischer Scientific, Rockford, IL).

\section{Analysis of fecal bacteria}

The samples were prepared according to a previously explained method. ${ }^{18}$ To analyze the microbes, the samples were inoculated onto agar plates with selective media and cultured aerobically for $24 \mathrm{~h}$ and anaerobically for $48 \mathrm{~h}$ at $37^{\circ} \mathrm{C}$, respectively, for Enterobacteriaceae (Desoxycholate agar) and Bifidobacteria (BL agar). Agar powders were purchased from MB cell, Seoul, Korea.

\section{Micro-CT analysis}

One week before the animal sacrifice, three representative mice from each group were chosen and anesthetized using Rumpun: Zoletil mixture, and micro-CT analysis was conducted according to a previously mentioned method. ${ }^{19}$

\section{Histological analysis}

For the histological analysis, colon tissues were cut longitudinally and fixed in $10 \%$ formalin. After embedding in paraffin, tissues were sectioned ( $5 \mu \mathrm{m}$ thick) and stained 
using hematoxylin and eosin. The stained sections were then examined using Axiophot Zeiss Z1 microscope (Carl Zeiss, Gottingen, Germany) at $1000 \times$ magnification.

\section{$R T-q P C R$ and Immunoblotting}

The changes in mRNA expression of genes associated with colon inflammation were assessed using RT-qPCR. After RNA extraction using the QIAGEN RNA easy mini kit (QIAGEN GmbH, Hilden, Germany), purity was confirmed. Further, using a cDNA reverse transcription kit (Applied Biosytems, CA), cDNA was synthesized, and PCR was performed. The primers used in this study are given in Table 2.

The protein levels of $\mathrm{p}-65$, phospho-p65, inducible nitric oxide synthase (iNOS), cyclooxygenase 2 (COX2), Zonula occludens-1 (ZO-1), and $\beta$-actin were determined using immunoblotting. The colon tissue was homogenized in RIPA lysis buffer (Pierce-Thermo Fisher Scientific Korea Ltd., Seoul, South Korea) containing $1 \%$ protease inhibitor and $1 \%$ phosphatase inhibitor cocktail (Merck, Seoul, South Korea) to obtain the protein extract. The protein was subjected to electrophoresis on an 8-10\% SDS-polyacrylamide gel, and then transferred to polyvinylidene difluoride membrane (Bio-Rad Laboratories, Hercules, CA).

Table 2. Primers Used in This Study

\begin{tabular}{|c|c|c|}
\hline $\begin{array}{l}\text { Gene } \\
\text { name }\end{array}$ & Primers & Sequence $\left(5^{\prime}-3^{\prime}\right)$ \\
\hline \multirow[t]{2}{*}{$N F-\kappa \mathrm{B}$} & Forward & GAA GTG AGA GAG TGA GCG AGA GAG \\
\hline & Reverse & CGG GTG GCG AAA CCT CCT C \\
\hline \multirow[t]{2}{*}{$T N F-\alpha$} & Forward & ACG GCA TGG ATC TCA AAG AC \\
\hline & Reverse & GTG GGT GAG GAG CAC GTA GT \\
\hline \multirow[t]{2}{*}{$I L-6$} & Forward & AAC GAT GAT GCA CTT GCA GA \\
\hline & Reverse & GAG CAT TGG AAA TTG GGG TA \\
\hline \multirow[t]{2}{*}{$I L-1 \beta$} & Forward & GAC CTT CCA GGA TGA GGA CA \\
\hline & Reverse & AGC TCA TAT GGG TCC GAC AG \\
\hline \multirow[t]{2}{*}{$i N O S$} & Forward & ATG TCC GAA GCA AAC ATC AC \\
\hline & Reverse & TAA TGT CCA GGA AGT AGG TG \\
\hline \multirow[t]{2}{*}{$I L-10$} & Forward & TAC CTG GTA GAA GTG ATG CC \\
\hline & Reverse & CAT CAT GTA TGC TTC TAT GC \\
\hline \multirow[t]{2}{*}{$F 4 / 80$} & Forward & TCC AGC ACA TCC AGC CAA AGC \\
\hline & Reverse & CCT CCA CTA GCA TCC AGA AGA AGC \\
\hline \multirow[t]{2}{*}{$M C P-1$} & Forward & TTC CTC CAC CAC CAT GCA G \\
\hline & Reverse & CCA GCC GGC AAC TGT GA \\
\hline \multirow[t]{2}{*}{ Claudin-1 } & Forward & TCT ACG AGG GAC TGT GGA TG \\
\hline & Reverse & TCA GAT TCA GCA AGG AGT CG \\
\hline \multirow[t]{2}{*}{$\mathrm{ZO}-1$} & Forward & ACC CGA AAC TGA TGC TGT GGA TAG \\
\hline & Reverse & AAA TGG CCG GGC AGA ACT TGT GTA \\
\hline \multirow[t]{2}{*}{ Mucl } & Forward & CTG TTC ACC ACC ACC ATG AC \\
\hline & Reverse & CTT GGA AGG GCA AGA AAA CC \\
\hline \multirow[t]{2}{*}{ Muc3 } & Forward & CCA CCA CTG TTG AAG TCA CAA \\
\hline & Reverse & CAG AAC CCT CCG TTC ATA CAA \\
\hline \multirow[t]{2}{*}{ GPR120 } & Forward & AGA GGC TTA CGC TGA GCT TG \\
\hline & Reverse & TGG ATC AAG ATG AGG AGG \\
\hline \multirow[t]{2}{*}{$\beta$-actin } & Forward & AGC CTT CCT TCT TGG GTA TGG \\
\hline & Reverse & CAC TTG CGG TGC ACG ATG GAG \\
\hline
\end{tabular}

GPR, G-protein receptor; IL, interleukin; iNOS, inducible nitric oxide synthase; MCP, monocyte chemoattractant protein; Muc, mucin; NF- $\kappa \mathrm{B}$, nuclear factor-kappa B; TNF, tumor necrosis factor; ZO, Zonula occludens-1.

\section{Statistical analysis}

The one-way analysis of variance (ANOVA) method of SPSS (version 17.0; SPSS, Inc., Chicago) was used to analyze statistical differences. Differences with $P<.05$ by Duncan's test were considered to be statistically significant. The values with different superscripts such as $a, b$, and $c$ indicate significant differences among the groups. Data are expressed as the means \pm standard deviations.

\section{RESULTS}

\section{PO improved HD-induced obesity}

As shown in Figure 1, the final body weight showed significant differences between the ND- and HD-fed groups. Although there was no significant difference between feed intakes among HD groups, the ND group had a significantly higher $(P<.05)$ feed intake compared with the HD-fed groups. The supplementation with FO and PO suppressed body weight gain compared with the HD group (Table 3 ). The liver and epididymal fat weights were also reduced in HDFO and HDPO groups $(P<.05)$. The micro-CT analysis of mice revealed that abdominal fat was reduced in HDFO and HDPO groups as shown in Figure 1D. The levels of serum TG and TC were also improved by the supplementation with FO and PO compared with the HD group $(P<.05)$ (Table 3).

\section{PO improved serum endotoxin levels and prevented $H D$-induced alteration in gut microbial populations}

As shown in Figure 2, treatment with FO and PO significantly reduced $(P<.05)$ the serum endotoxin levels compared with HD. The number of Bifidobacteria was reduced, whereas the number of Enterobacteriaceae was increased in the HD group. However, in both the HDFO and HDPO groups, the number of Bifidobacteria was increased, whereas that of Enterobacteriaceae was decreased $(P<.05)$.

\section{$P O$ improved the HD-induced inflammation in the colon}

As shown in Figure 3A, B, the consumption of HD increased the macroscopic score and reduced the length of the colon compared with the ND group. Interestingly, the HDFO and HDPO groups retained the colon length similar to that of ND $(P<.05)$. The $\mathrm{H} \& \mathrm{E}$ staining of the colon revealed that the endothelia of HD-fed groups were disrupted (Fig. 3C). However, treatment with FO and PO suppressed the disruption of colon epithelia, suggesting their potential effects on colon health.

Supplementation with FO and PO significantly reduced $(P<.05)$ the levels of proinflammatory cytokines, such as IL- $1 \beta$ and TNF- $\alpha$ in serum, compared with the HD group (Table 3). Similarly, the analysis of colon tissue revealed that HDFO and HDPO groups exhibited a significant reduction $(P<.05)$ in proinflammatory cytokine levels such as IL- $1 \beta$, IL-6, and TNF- $\alpha$, in comparison with HD (Fig. 4A). 

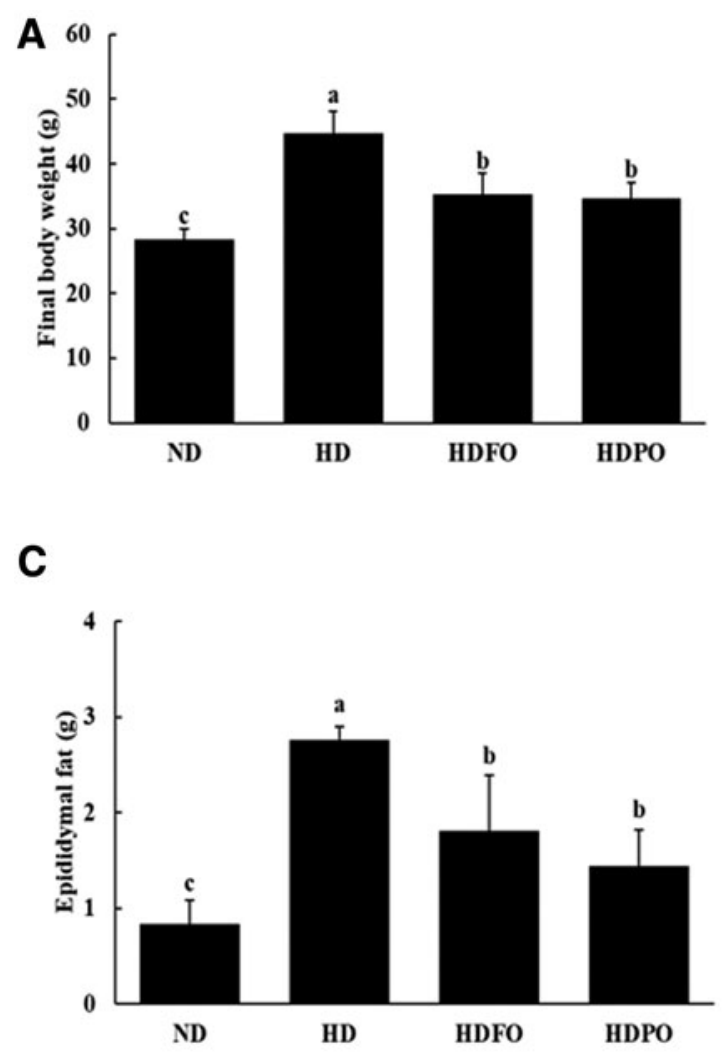
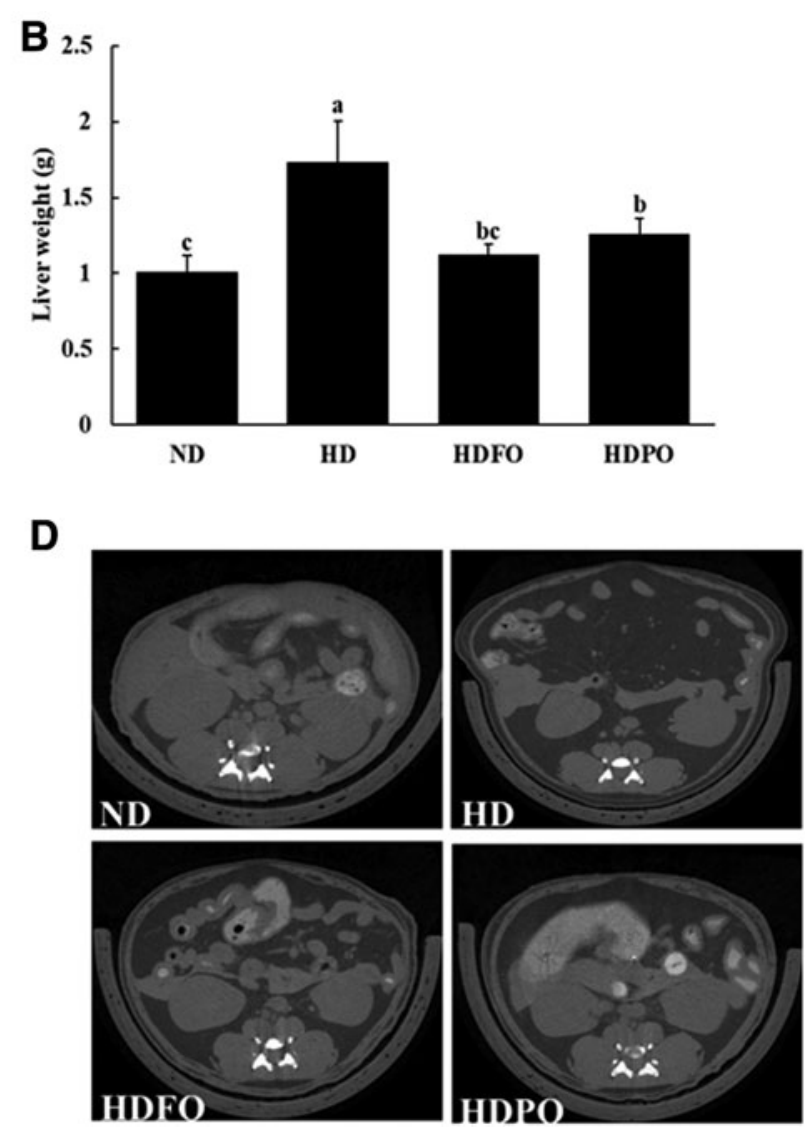

FIG. 1. Effect of PO on anthropometric parameters in experimental mice: (A) body weight, (B) liver weight, (C) epididymal fat weight, and (D) micro-CT image of abdominal fat. Values are expressed as mean $\pm \mathrm{SD}, n=8$. Values with different superscripts (a, b, c) are significantly different among groups by ANOVA with Duncan's multiple range test at $P<.05$. The darker grey regions in micro-CT image indicate fat. ND, $10 \% \mathrm{kcal}$ from fat; HD, $60 \% \mathrm{kcal}$ from fat; HDFO, $60 \% \mathrm{kcal}$ from fat diet supplemented with FO; HDPO, 60\% kcal from fat diet supplemented with PO. The supplemented group consumed a diet with a final composition of $8 \%$ oil in it. ANOVA, analysis of variance; FO, fish oil; HD, highfat diet; HDFO, high-fat diet supplemented with FO; HDPO, high-fat diet supplemented with PO; ND, normal diet; PO, perilla oil; SD, standard deviation.

As shown in Figure 4B, the gene expression of proinflammatory markers such as $I L-6, I L-1 \beta$, and $T N F-\alpha$ was significantly lower $(P<.05)$ in HDFO and HDPO compared with the HD group. However, the mRNA expression level of $I L-10$, an anti-inflammatory marker, and $\mathrm{ZO}-1$, a tight junction protein, was significantly higher $(P<.05)$ in the
FO- and PO-treated groups compared with HD group. Claudin-1, another tight junction protein, showed an increasing tendency in FO and PO, but was not significantly higher compared with HD. Furthermore, the expression of membrane-associated colon mucins, Mucl and Muc3, was upregulated in HDFO and HDPO groups compared with the

Table 3. Body Weight Gain, Feed Intake, Serum Lipids, and Proinflammatory Cytokine Levels

\begin{tabular}{|c|c|c|c|c|}
\hline Parameters & $N D$ & $H D$ & $H D F O$ & $H D P O$ \\
\hline Body weight gain (g) & $4.47 \pm 2.22^{\mathrm{c}}$ & $21.36 \pm 3.66^{\mathrm{a}}$ & $11.80 \pm 3.72^{\mathrm{b}}$ & $10.50 \pm 2.24^{\mathrm{b}}$ \\
\hline Feed intake (g/mice/day) & $4.27 \pm 0.51^{\mathrm{a}}$ & $2.43 \pm 0.13^{b}$ & $2.58 \pm 0.28^{\mathrm{b}}$ & $2.75 \pm 0.009^{b}$ \\
\hline Triglyceride (mg/dL) & $24.27 \pm 10.06^{\mathrm{c}}$ & $64.40 \pm 14.89^{\mathrm{a}}$ & $36.84 \pm 11.78^{\mathrm{bc}}$ & $46.84 \pm 11.06^{\mathrm{b}}$ \\
\hline Total cholesterol (mg/dL) & $44.58 \pm 20.45^{\mathrm{c}}$ & $124.64 \pm 16.27^{\mathrm{a}}$ & $73.20 \pm 45.81^{\mathrm{bc}}$ & $108.13 \pm 15.26^{\mathrm{ab}}$ \\
\hline HDL (mg/dL) & $62.55 \pm 8.16^{\mathrm{b}}$ & $110.36 \pm 15.87^{\mathrm{a}}$ & $91.42 \pm 13.44^{\mathrm{a}}$ & $97.14 \pm 20.71^{\mathrm{a}}$ \\
\hline TNF- $\alpha(\mathrm{pg} / \mathrm{mL})$ & $34.81 \pm 4.14^{\mathrm{b}}$ & $51.00 \pm 14.18^{\mathrm{a}}$ & $11.70 \pm 1.74^{\mathrm{c}}$ & $13.97 \pm 3.59^{c}$ \\
\hline $\mathrm{IL}-1 \beta(\mathrm{pg} / \mathrm{mL})$ & $35.90 \pm 1.53^{\mathrm{b}}$ & $46.70 \pm 11.69^{\mathrm{a}}$ & $36.27 \pm 1.06^{\mathrm{b}}$ & $35.31 \pm 0.73^{\mathrm{b}}$ \\
\hline
\end{tabular}

\footnotetext{
Values are expressed as mean $\pm \mathrm{SD}, n=8$. Values with different superscripts ( $\mathrm{a}, \mathrm{b}, \mathrm{c}$ ) are significantly different among groups by ANOVA with Duncan's multiple range test at $P<.05$. ND, $10 \% \mathrm{kcal}$ from fat; HD, 60\% kcal from fat; HDFO, 60\% kcal from fat diet supplemented with FO; HDPO, 60\% kcal from fat diet supplemented with PO. The supplemented group consumed a diet with a final composition of $8 \%$ oil in it.
}

ANOVA, analysis of variance; SD, standard deviation. 


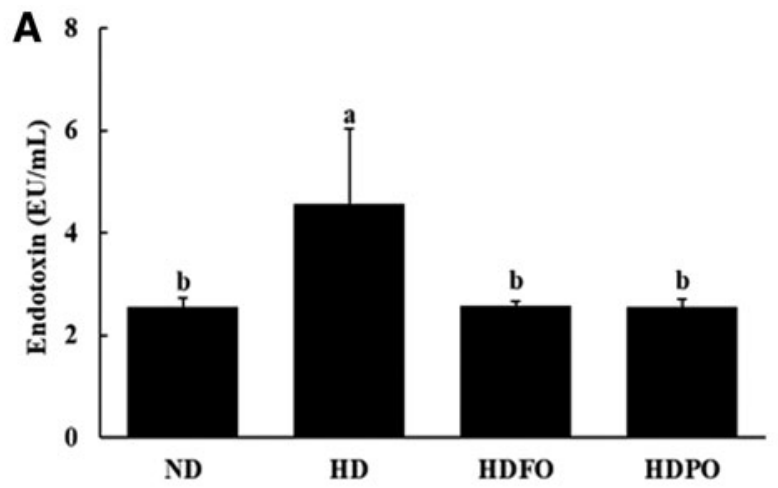

B
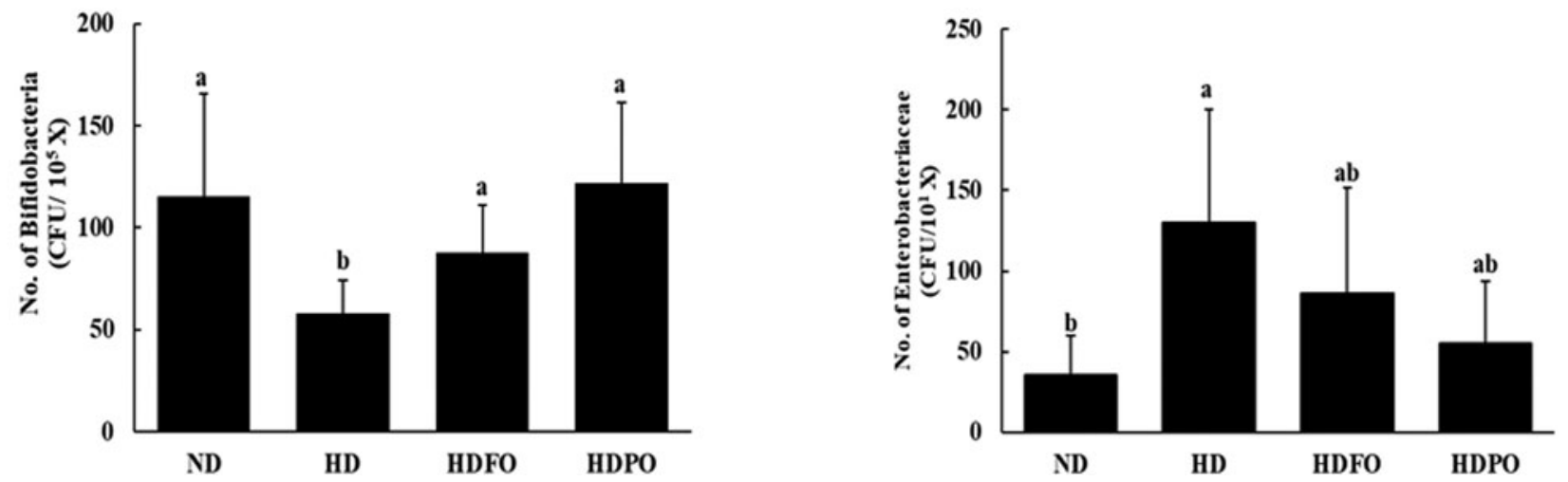

FIG. 2. Serum endotoxin levels (A) and the number of fecal Bifidobacteria and Enterobacteriaceae (B) in experimental mice. Values are expressed as mean $\pm \mathrm{SD}, n=8$. Values with different superscripts $(\mathrm{a}, \mathrm{b}, \mathrm{c})$ are significantly different among groups by ANOVA with Duncan's multiple range test at $P<.05$. ND, $10 \%$ kcal from fat; HD, $60 \%$ kcal from fat; HDFO, $60 \%$ kcal from fat diet supplemented with FO; HDPO, $60 \%$ kcal from fat diet supplemented with PO. The supplemented group consumed a diet with a final composition of $8 \%$ oil in it.

A
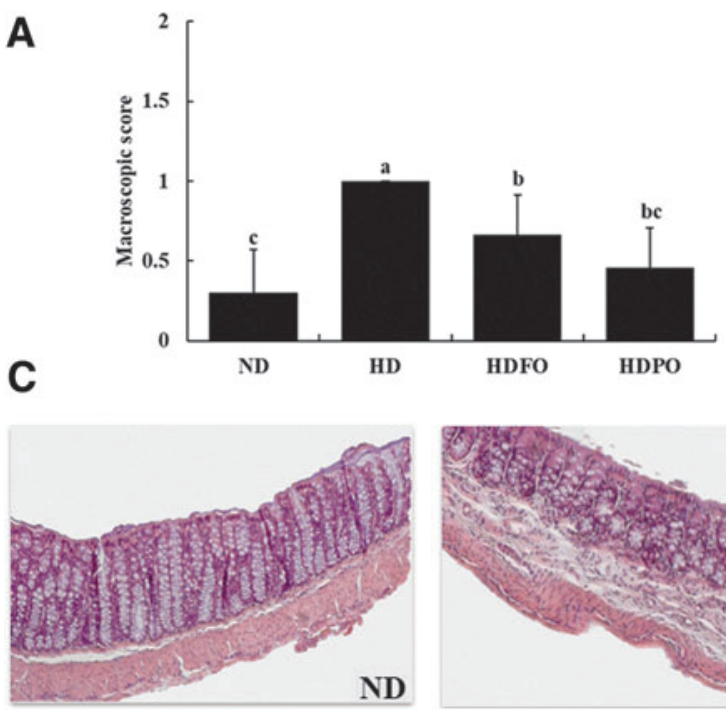
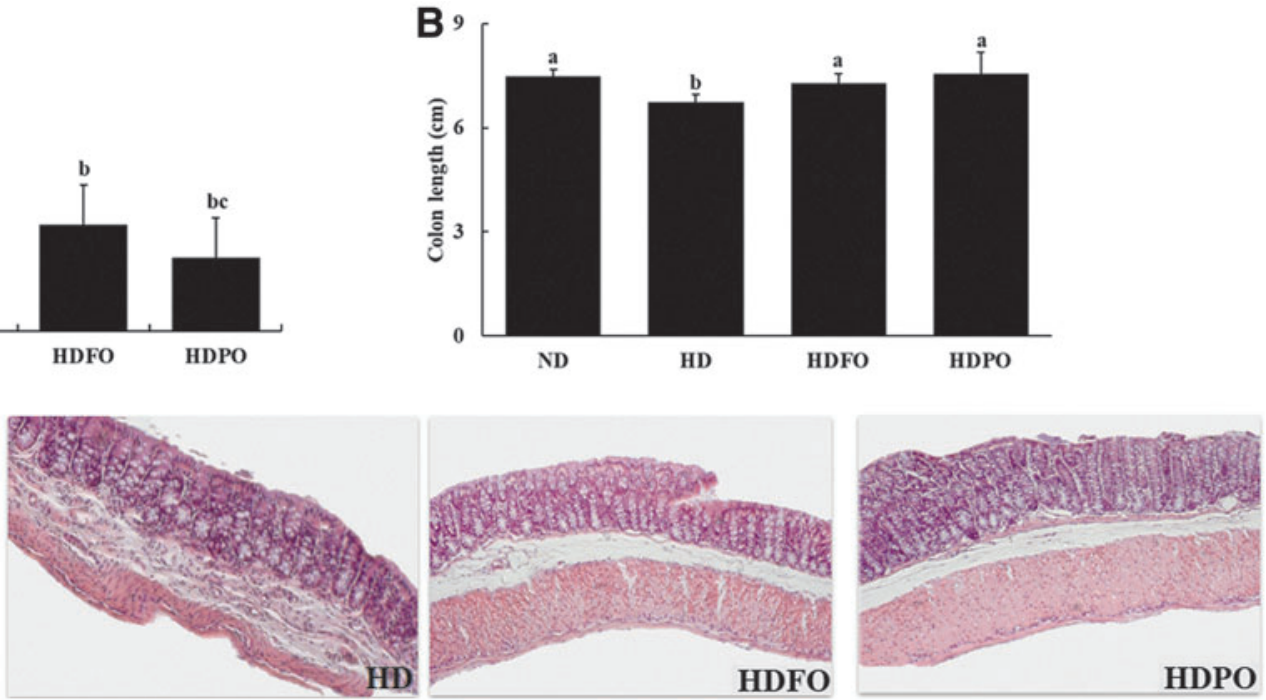

FIG. 3. (A) Macroscopic score, (B) colon length, and (C) H \& E staining of the colon in experimental mice. Values are expressed as mean \pm SD, $n=8$. Values with different superscripts (a, b, c) are significantly different among groups by ANOVA with Duncan's multiple range test at $P<.05$. ND, $10 \%$ kcal from fat; HD, $60 \%$ kcal from fat; HDFO, $60 \%$ kcal from fat diet supplemented with FO; HDPO, 60\% kcal from fat diet supplemented with PO. The supplemented group consumed a diet with a final composition of $8 \%$ oil in it. Color images are available online. 
A

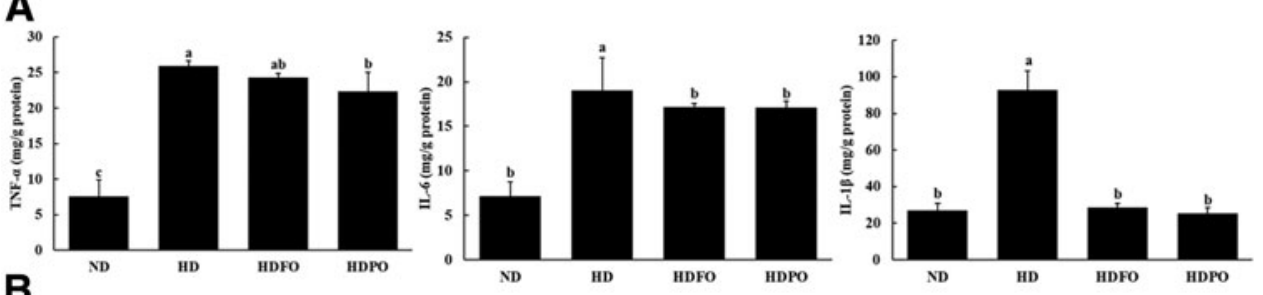

$\mathbf{B}_{4}$

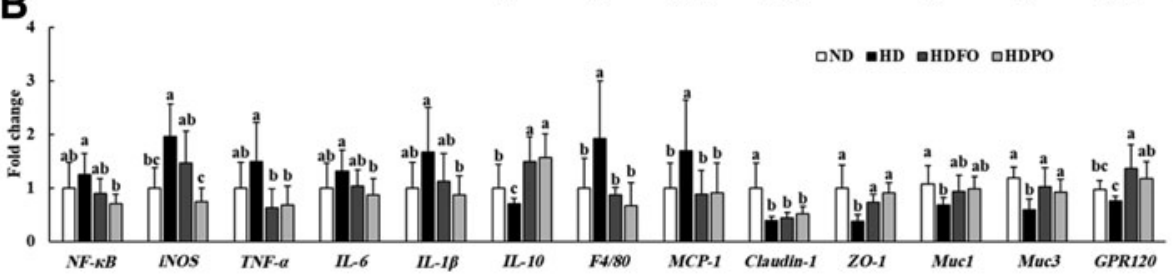

C
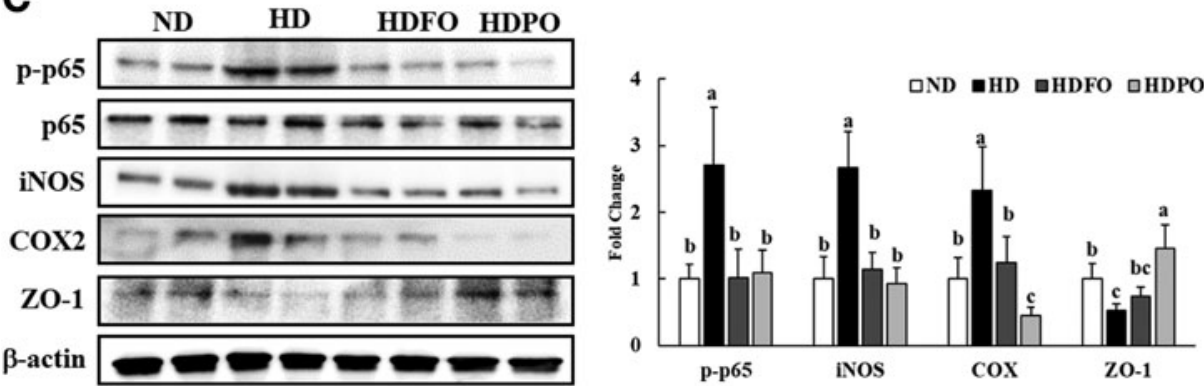

FIG. 4. (A) Proinflammatory cytokine levels, (B) mRNA expression levels, and (C) protein expression in the colon of experimental mice. Values are expressed as mean $\pm \mathrm{SD}, n=8$. Values with different superscripts $(\mathrm{a}, \mathrm{b}, \mathrm{c})$ are significantly different among groups by ANOVA with Duncan's multiple range test at $P<.05$. $\mathrm{ND}, 10 \% \mathrm{kcal}$ from fat; HD, $60 \% \mathrm{kcal}$ from fat; HDFO, $60 \%$ kcal from fat diet supplemented with FO; HDPO, $60 \%$ kcal from fat diet supplemented with PO. The supplemented group consumed a diet with a final composition of $8 \%$ oil in it.

HD group, showing that treatment with FO and PO improved colon inflammatory conditions and enhanced intestinal barrier functions. The mRNA expression of two markers of macrophage infiltration, F4/80 and monocyte chemoattractant protein-1 ( $M C P-1)$, was also significantly lower $(P<.05)$ in FO- and PO-treated groups compared with the $\mathrm{HD}$ group. In addition, the mRNA expression of $\mathrm{G}$ protein-coupled receptor 120 (GPR120), the cell membrane protein receptor activated by long chain fatty acids, was higher in FO- and PO-treated groups.

The immunoblot analysis of colon tissue revealed that the levels of phosphorylated p65 (p-p65) (activated subunit of $\mathrm{NF}-\kappa \mathrm{B}$ ) increased with HD consumption, whereas HDFO and HDPO showed lower levels $(P<.05)$ of p-p65 compared with the HD group (Fig. 4C). The expression of iNOS and COX2 was also reduced in FO- and PO-treated groups $(P<.05)$. In addition, the expression of the tight junction protein, ZO-1, was upregulated in FO- and PO-treated groups compared with the HD group $(P<.05)$. Interestingly, PO showed higher expression of ZO-1 compared with FO.

\section{DISCUSSION}

Prolonged consumption of a HD has been linked to the initiation or exacerbation of various disease conditions including IBD. ${ }^{20}$ Damaged intestinal epithelia and mucosa infiltrated with activated immune cells that produce excessive cytokines and the upregulation of inflammatory proteins are associated with colitis. In patients with IBDs, the levels of proinflammatory cytokines are reported to be higher compared with those of healthy individuals. ${ }^{21}$ Therefore, changes in dietary patterns that include the consumption of natural anti-inflammatory agents can be effective for preventing the exacerbation of inflammationassociated complications. Omega-3 fatty acids have several health benefits including protective effect against inflammatory conditions. ${ }^{22}$ Several studies have reported the role of HDs in the aggravation of chemically induced colitis. ${ }^{23-25}$ However, studies on the association of HDs and colitis in normal conditions are very limited. ${ }^{26,27}$ In addition, the effects of omega-3 fatty acids, especially in edible forms, such as oils, on HD-induced colitis have not yet been investigated. Therefore, the aim of this study was to investigate the effects of PO (a rich source of omega- 3 fatty acids) and FO (widely studied source of omega-3 fatty acids) on HD-induced colon inflammation.

Similar to previous reports, the fatty acid composition analysis of PO used in our study showed that $\alpha$-linolenic acid was in the highest amount (59.5\%, omega-3 fatty acid), followed by oleic acid (14.2\%, monounsaturated fatty acid), linoleic acid (12.3\%, omega-6 fatty acid), and palmitic acid (5.8\%, saturated fatty acid). ${ }^{28,29}$ The main fatty acids present in FO used in this study were EPA (15\%, omega-3 fatty acid), DHA (15\%, omega-3 fatty acid), palmitic acid (20\%, saturated fatty acid), palmitoleic acid (14\%, monounsaturated fatty acid), oleic acid (12\%, monounsaturated fatty acid), linoleic acid ( $<3 \%$, omega- 6 fatty acid), and linolenic acid $(<3 \%$, omega-3 fatty acid).

Treatment with PO improved HD-induced body weight gain, ectopic fat accumulation-associated organ weight 
gains, and serum lipid levels. A recent report by Chen $e t$ al. showed that PO reversed the HD-mediated increase in serum lipids and hepatic lipids through the enhanced excretion of cholesterol and bile acid through feces. ${ }^{30}$ A previous study showed that both FO and PO effectively reduced TC levels, whereas only FO reduced the TG levels in the serum of Wister rats fed with a HD. ${ }^{31}$ However, in this study both FO and PO attenuated the HD-induced rise in serum TG levels. The results not only confirmed that omega-3-rich oils can exert antiobesity effects but also showed that PO exhibited similar effects to those of FO.

Studies in human subjects and animal models have shown that obesity is characterized by a decrease in the phylum Bacteroidetes and increase in phylum Firmicutes in the intestinal microbiota. Kim et al. previously showed that the consumption of $\mathrm{HD}$ provides a favorable condition for the growth of gram-negative bacteria, such as Enterobacteriaceae, which may contribute to increased endotoxin production in the intestine. ${ }^{4}$ Treatment with $\mathrm{PO}$ and FO reduced the endotoxin levels and significantly increased $(P<.05)$ the number of Bifidobacteria, while showing a tendency to decrease the number of Enterobacteriaceae, suggesting that omega-3 fatty-acid-rich oils improve HDassociated changes in gut microbial population.

Although adipose tissue is the primary site of HD-induced inflammation, recent studies have shown that inflammation also occurs in the gastrointestinal tract as a result of HD consumption. ${ }^{4}$ The destruction of colon crypts and infiltration of macrophages to the mucosa cause the disruption of typical mucosal architecture that results in the shortening and narrowing of the colon. ${ }^{32}$ In this study, the consumption of HD for 16 weeks increased the macroscopic score and caused the shortening of the colon. Supplementation with PO and FO retained the colon conditions similar to ND. In addition, the levels of proinflammatory cytokines in the colon and serum were reduced in the HDFO and HDPO groups. TNF- $\alpha$ is considered to have a major role in the pathogenesis of IBD, not only in initiating cytotoxic, apoptotic, and acute-phase responses but also in increasing the production of other proinflammatory cytokines, such as IL$1 \beta$ and IL-6. In inflammatory conditions, IL- $1 \beta$ initiates the production and activation of COX2 and iNOS, and IL-6 activates NF- $\kappa$ B in endothelial cells. ${ }^{33}$

LPS and TNF- $\alpha$ activate the NF- $\kappa$ B pathway, which further activates inflammatory genes that have a major role in the pathogenesis of inflammatory diseases. ${ }^{34}$ Omega-3 fatty acids inhibited NF- $\kappa$ B activation and, thus, downregulated the expression of proinflammatory genes in the THP-1 macrophage cell line. ${ }^{35}$ Interestingly, FO and PO prevented NF- $\kappa \mathrm{B}$ activation in colon tissue and its downstream targets, showing that both of the omega-3-rich oils reduced the inflammatory conditions in colon. In addition, treatment with FO and PO increased Muc1 and Muc3, which enhance the mucosal barrier defense against infection and reimburse wounded epithelium, respectively. Tight junction proteins play an integral role in maintaining cell polarity and paracellular transports, and also act as a physical barrier against pathogens and harmful molecules providing pro- tection to the body against inflammation or infections. ${ }^{36}$ TNF- $\alpha$ causes intestinal tight junction permeability through NF- $\kappa$ B activation, simultaneous downregulation and alteration of localization of ZO-1 proteins. ${ }^{37}$ Furthermore, an increase in LPS levels causes changes in tight junction proteins and alters the permeability of the intestine, which increases systemic low-grade inflammation in obesity. ${ }^{38}$ The mRNA and protein expression of ZO- 1 was upregulated in the HDFO and HDPO groups compared with the HD group, suggesting that PO and FO inhibited LPS- and TNF- $\alpha$-associated NF- $\kappa$ B activation, thus preventing the changes in tight junction protein ZO-1. This in turn maintains the colon epithelial integrity and prevents meta-inflammation.

Activation of GPR120 by omega-3 fatty acids has been shown to have anti-inflammatory activity in cells, such as macrophages, adipocytes, Kupffer cells, and neurons. ${ }^{39}$ GPR120 activation reduces the transforming growth factor beta-activated kinase 1 (TAK1) activation by LPS or TNF- $\alpha$, which in turn inhibits the activation of NF- $\kappa \mathrm{B}$, the major inflammatory signaling pathway. A previous study showed that GPR120 acts as an anti-inflammatory mediator in the chronic colitis model of IL-10 knockout mice. ${ }^{40}$ In our study, PO and FO significantly $(P<.05)$ increased the expression of GPR120 compared with the HD group, suggesting that the omega-3 fatty acids present in the oils exerted the anti-inflammatory properties in the colon, possibly through the stimulation of the GPR 120 receptor.

In conclusion, our study revealed that PO ameliorates HD-induced colon inflammation by improving the macroscopic score and colon length, reducing proinflammatory cytokines, improving the intestinal barrier protection, inhibiting the NF- $\kappa$ B pathway, and reducing the expression of proinflammatory genes, which might be mediated through the activation of GPR120. To the best of our knowledge, this is the first report on protective effect of PO against HDinduced colon inflammation, and we used edible oil forms to conduct the experiments. We showed that diet modifications that include consumption of PO, omega-3 fatty-acid-rich oil, could contribute to resolving HD-induced complications such as ulcerative colitis. Interestingly, the effect of PO, which is a popular edible oil in Korea, was similar to FO, a well-known omega-3 fatty acid supplement, confirming both the health benefits of using PO in the Korean cuisine and the potential of PO as an alternative omega-3 supplement.

\section{ACKNOWLEDGMENT}

The authors thank Yu-Jung Chang, $\mathrm{PhD}$, at the Jeonbuk National University Center for University-Wide Research Facilities (CURF) for helping in micro-CT.

\section{AUTHOR DISCLOSURE STATEMENT}

No competing financial interests exist.

\section{FUNDING INFORMATION}

This research was funded by the National Research Foundation of Korea (NRF) grant funded by the Korea government (Ministry of Education) (2017R1D1A1B03029418). 


\section{SUPPLEMENTARY MATERIAL}

Supplementary Table S1

\section{REFERENCES}

1. Minihane AM, Vinoy S, Russell WR, et al.: Low-grade inflammation, diet composition and health: Current research evidence and its translation. Br J Nutr 2015;114:999-1012.

2. $\mathrm{Ng} \mathrm{Sc}$, Shi HY, Hamidi N, et al.: Worldwide incidence and prevalence of inflammatory bowel disease in the 21st century: A systematic review of population-based studies. Lancet 2017;390: 2769-2778.

3. Kwak MS, Cha JM, Lee HH, et al.: Emerging trends of inflammatory bowel disease in South Korea: A nationwide populationbased study. J Gastroenterol Hepatol 2019;34:1018-1026.

4. Kim KA, Gu W, Lee IA, Joh EH, Kim, DH: High fat dietinduced gut microbiota exacerbates inflammation and obesity in mice via the TLR4 signaling pathway. PLoS One 2012;7:e47713.

5. Swanson D, Block R, Mousa, SA: Omega-3 fatty acids EPA and DHA: Health benefits throughout life. Adv Nutr 2012;3:1-7.

6. Ander BP, Dupasquier CM, Prociuk MA, Pierce GN: Polyunsaturated fatty acids and their effects on cardiovascular disease. Exp Clin Cardiol 2003;8:164-172.

7. D'Archivio M, Scazzocchio B, Vari R, Santangelo C, Giovannini C, Masella, RJ: Recent evidence on the role of dietary PUFAs in cancer development and prevention. Curr Med Chem 2018;25: $1818-1836$.

8. Bargut TCL, Ferreira TPT, Daleprane JB, Martins MA, Silva PMR, Aguila MB: Fish oil has beneficial effects on allergeninduced airway inflammation and hyperreactivity in mice. PLoS One 2013;8:e75059.

9. de Arruda LLM, Ames FQ, de Morais DR, et al.: A single administration of fish oil inhibits the acute inflammatory response in rats. Asian Pac J Trop Med 2017;10:765-772.

10. Li Y, Zhao F, Wu Q, et al.: Fish oil diet may reduce inflammatory levels in the liver of middle-aged rats. Sci Rep 2017;7: 6241.

11. Bouwens M, van de Rest O, Dellschaft N, et al.: Fish-oil supplementation induces antiinflammatory gene expression profiles in human blood mononuclear cells. Am J Clin Nutr 2009;90: 415-424.

12. Ezaki O, Takahashi M, Shigematsu T, et al.: Long-term effects of dietary alpha-linolenic acid from perilla oil on serum fatty acids composition and on the risk factors of coronary heart disease in Japanese elderly subjects. J Nutr Sci Vitaminol 1999;45:759-772.

13. Asif M: Health effects of omega-3,6,9 fatty acids: Perilla frutescens is a good example of plant oils. Orient Pharm Exp Med 2011;11:51-59.

14. Cha Y. Jang JY, Ban YH, et al.: Anti-atherosclerotic effects of perilla oil in rabbits fed a high-cholesterol diet. Lab Anim Res 2016;32:171-179.

15. Wei M, Xiong $\mathrm{P}$, Zhang L, et al.: Perilla oil and exercise decrease expressions of tumor necrosis factor-alpha, plasminogen activator inhibitor-1 and highly sensitive C-reactive protein in patients with hyperlipidemia. J Tradit Chin Med 2013;33:170-175.

16. Chang HH, Chen CS, Lin JY: Protective effect of dietary perilla oil on allergic inflammation in asthmatic mice. Eur J Lipid Sc Technol 2012;114:1007-1015.
17. Yang W, Chen X, Liu Y, et al: N-3 polyunsaturated fatty acids increase hepatic fibroblast growth factor 21 sensitivity via a PPAR- $\gamma$ - $\beta$-klotho pathway. Mol Nutr Food Res 2017;61: 1601075.

18. Kim KA, Jang SE, Jeong JJ, Yu DH, Han MJ, Kim DH: Doenjang, a Korean soybean paste, ameliorates TNBS-induced colitis in mice by suppressing gut microbial lipopolysaccharide production and NF- $\kappa$ B activation. $J$ Funct Foods 2014;11:417-427.

19. Thomas SS, Kim M, Lee SJ, Cha YS: Antiobesity effects of purple Perilla (Perilla frutescens var. acuta) on adipocyte differentiation and mice fed a high-fat diet. J Food Sci 2018;83: 2384-2393.

20. Duan Y, Zeng L, Zheng C, et al.: Inflammatory links between high fat diets and diseases. Front Immunol 2018;9:2649.

21. Paik J, Fierce Y, Treuting PM, Brabb T, Maggio-Price L: Highfat diet-induced obesity exacerbates inflammatory bowel disease in genetically susceptible Mdr1a-/- male mice. J Nutr 2013;143: 1240-1247.

22. Fahrmann JF, Ballester OF, Ballester G, et al:: Inhibition of nuclear factor kappa B activation in early-stage chronic lymphocytic leukemia by omega-3 fatty acids. Cancer Invest 2013; 31:24-38.

23. Teixeira LG, Leonel AJ, Aguilar EC, et al: The combination of high-fat diet-induced obesity and chronic ulcerative colitis reciprocally exacerbates adipose tissue and colon inflammation. Lipids Health Dis 2011;10:204.

24. Cheng L, Jin H, Qiang Y, et al.: High fat diet exacerbates dextran sulfate sodium induced colitis through disturbing mucosal dendritic cell homeostasis. Int Immunopharmacol 2016;40:1-10.

25. Lee JC, Lee HY, Kim TK, et al.: Obesogenic diet-induced gut barrier dysfunction and pathobiont expansion aggravate experimental colitis. PLoS One 2017;12:e0187515.

26. Lim SM, Choi HS, Kim DH: The mixture of Anemarrhena asphodeloides and Coptis chinensis attenuates high-fat diet-induced colitis in mice. Am J Chin Med 2017;45:1033-1046.

27. Lim SM, Kim DH: Bifidobacterium adolescentis IM38 ameliorates high-fat diet-induced colitis in mice by inhibiting NF- $\kappa \mathrm{B}$ activation and lipopolysaccharide production by gut microbiota. Nutr Res 2017;41:86-96.

28. Longvah T, Deosthale YG: Chemical and nutritional studies on Hanshi (Perilla frutescens), a traditional oilseed from Northeast India. J Am Oil Chem Soc 1991;68:781-784.

29. Lee AY, Choi JM, Lee MH, Lee J, Lee S, Cho EJ: Protective effects of perilla oil and alpha linolenic acid on SH-SY5Y neuronal cell death induced by hydrogen peroxide. Nutr Res Pract 2018;12:93-100.

30. Chen T, Yuan F, Wang H, et al.: Perilla oil supplementation ameliorates high-fat/high-cholesterol diet induced nonalcoholic fatty liver disease in rats via enhanced fecal cholesterol and bile acid excretion. Biomed Res Int 2016;2016:2384561.

31. Tian Y, Wang H, Yuan F, et al.: Perilla oil has similar protective effects of fish oil on high-fat diet-induced nonalcoholic fatty liver disease and gut dysbiosis. Biomed Res Int 2016;2016:9462571.

32. DeRoche TC, Xiao SY, Liu X: Histological evaluation in ulcerative colitis. Gastroenterol Rep 2014;2:178-192.

33. Muzes G, Molnar B, Tulassay Z, Sipos F: Changes of the cytokine profile in inflammatory bowel diseases. World J Gastroenterol 2012;18:5848-5861.

34. Baker RG, Hayden MS, Ghosh S: NF-kappaB, inflammation, and metabolic disease. Cell Metab 2011;13:11-22. 
35. Allam-Ndoul B, Guénard F, Barbier O, Vohl MC: Effect of n-3 fatty acids on the expression of inflammatory genes in THP-1 macrophages. Lipids Health Dis 2016;15:69.

36. Lee B, Moon KM, Kim CY: Tight junction in the intestinal epithelium: Its association with diseases and regulation by phytochemicals. J Immunol Res 2018;2018:2645465.

37. Ma TY, Iwamoto GK, Hoa NT, et al.: TNF-alpha-induced increase in intestinal epithelial tight junction permeability requires NF-kappa B activation. Am J Physiol Gastrointest Liver Physiol 2004;286:G367-G376.
38. Yook JS, Kim KA, Kim M, Cha YS.: Black adzuki bean (Vigna angularis) attenuates high-fat diet-induced colon inflammation in mice. J Med Food 2017;20:367-375.

39. Ren Z, Chen L, Wang Y, et al.: Activation of the omega-3 fatty acid receptor GPR120 protects against focal cerebral ischemic injury by reventing inflammation and apoptosis in mice. J Immunol 2019;202:747-759.

40. Zhao J, Wang H, Shi P, Wang W, Sun Y: GPR120, a potential therapeutic target for experimental colitis in IL-10 deficient mice. Oncotarget 2017;8:8397-8405. 